\title{
DESEMPENHO PRODUTIVO DE JUVENIS DE PACU (Piaractus mesopotamicus) SUBMETIDOS A DIETAS SUPLEMENTADAS COM $\beta$-GLUCANO
}

\author{
Productive performance of Раси (Piaractus mesopotamicus) juveniles \\ submitted to diets supplemented with $\beta$-glucan
}

\section{Marianne Schorer ${ }^{[a]}$, João Batista Kochenborger Fernandes ${ }^{[b]}$, Elisabeth Criscuolo Urbinati ${ }^{[\mathrm{c}]}$, Ian Taibo Timpone ${ }^{[\mathrm{d}]}$}

\footnotetext{
[a] Zootecnista, Mestre em Aquicultura do Centro de Aquicultura da UNESP (Caunesp), Jaboticabal, SP - Brasil, e-mail: marianne_schorer@uol.com.br

[b] Zootecnista, Doutor, Pesquisador do Centro de Aquicultura da Universidade Estadual de São Paulo (Caunesp), Jaboticabal, SP - Brasil, e-mail: jbatista@caunesp.unesp.br

[c] Biomédica, professora Doutora do Departamento de Morfologia e Fisiologia Animal da Faculdade de Ciências Agrárias e Veterinárias e Centro de Aquicultura da Universidade Estadual de São Paulo (Caunesp), Jaboticabal, SP - Brasil, e-mail: bethurb@caunesp.unesp.br

[d]Zootecnista, Mestre em Zootecnia da Universidade Estadual de São Paulo (UNESP), Jaboticabal, SP - Brasil, e-mail: iantimpone@hotmail.com
}

\begin{abstract}
Resumo
O emprego do $\beta$-glucano na alimentação de peixes é cada vez maior, por aumentar a resistência às doenças e por promover benefícios ao trato gastrointestinal, resultando em melhor desempenho produtivo. Este trabalho teve como objetivo avaliar a utilização do prebiótico $\beta$-glucano em rações peletizadas e extrusadas no desempenho produtivo de juvenis de pacu (Piaractus mesopotamicus). Foram utilizados 640 juvenis de pacu $(24,7 \pm 2,0 \mathrm{~g})$ distribuídos em 32 aquários de vidro com capacidade volumétrica de 130 litros (20 peixes/aquário). Durante todo o período experimental, a água permaneceu a uma temperatura de $26,25^{\circ} \mathrm{C}$ e os parâmetros limnológicos (oxigênio dissolvido, $\mathrm{pH}$, alcalinidade e amônia total permaneceram dentro dos níveis adequados para a espécie. O delineamento utilizado foi inteiramente casualizado em esquema fatorial $2 \times 4$, com dois processamentos das dietas (extrusadas e peletizadas), quatro níveis de inclusão do $\beta$-glucano nas rações: 0 (controle), $0,1 \%, 0,2 \%$ e $0,3 \%$, e com quatro repetições. Os dados de ganho de peso, peso final, taxa de crescimento específico, consumo e conversão alimentar foram coletados e avaliados durante 90 dias. Os resultados obtidos mostraram que os níveis de $\beta$-glucano avaliados e os diferentes tipos de processamento da dieta não afetaram o desempenho produtivo de juvenis de pacu. Apesar de não significativo, os peixes alimentados com o nível de $0,3 \%$ de $\beta$-glucano apresentaram maior ganho de peso, taxa de crescimento específico e peso final.
\end{abstract}

Palavras-chave: Pacu. Imunoestimulantes. Processamento de ração.

Rev. Acad., Ciênc. Agrár. Ambient., Curitiba, v. 7, n. 4, p. 433-443, out./dez. 2009 


\begin{abstract}
The use of $\beta$-glucan in the diet of fish is increased, and promotes resistance to diseases and benefits the gastrointestinal tract, resulting in better performance. This study aimed to evaluate the use of prebiotic $\beta$-glucan in extruded and pelleted diets on performance of juvenile pacu (Piaractus mesopotamicus). Were used 640 pacu juveniles $(24.7 \pm 2.0 \mathrm{~g}$ ) distributed in 32 aquariums with capacity of volume 130 liters (20 fish/aquaria). Throughout the trial period, the water remained at a temperature of $26.25^{\circ} \mathrm{C}$ and limnological parameters (dissolved oxygen, $\mathrm{pH}$, alkalinity and total ammonia remained within the appropriate levels for the species. The design was completely randomized in a factorial $2 \times 4$ with two cases of diets (extruded and pelleted), with four levels of inclusion of $\beta$-glucan in the feed: 0 (control), $0.1 \%, 0.2 \%, 0.3 \%$, and with four replications. The data of weight gain, final weight, specific growth rate and consumption were collected and evaluated for 90 days. The results showed that levels of $\beta$-glucan and different types of processing of the diet did not affect the performance of pacu juveniles. While not significant, the fish fed with $0,3 \%$ levels of $\beta$-glucan had greater values of weight gain, specific growth rate and final weight.
\end{abstract}

Keywords: Pacu. Immunostimulants. Feed processing.

\title{
INTRODUÇÃO
}

Atualmente as atividades agropecuárias têm tido grande destaque na economia mundial, entre elas a produção de peixes, que apresenta índices de crescimento superiores às demais atividades rurais (VIEIRA et al., 2005). Em consequência, há um maior interesse em se investir na nutrição dos animais, visando a um melhor desempenho produtivo e econômico na criação.

A aquicultura no Brasil tem sido desenvolvida muito modestamente, se comparada com outras partes do mundo, onde ocupa um lugar de destaque como produtora de produtos de exportação por excelência. Isso se dá, principalmente, devido à falta de uma política setorial que priorize linhas de apoio governamental à produção e à necessidade de uma definição das alternativas de maior impacto sócio-econômico com vistas ao aproveitamento das potencialidades naturais de cada região (CAMARGO; POUEY, 2005). O Brasil ocupa a $20^{a}$ posição mundial entre os produtores de pescado cultivado (FAO, 2003).

A produção de peixes no Brasil ainda apresenta resultados modestos de desenvolvimento, devido aos processos de produção adotados e à falta de informação sobre espécies nativas com potencial zootécnico (PRIETO et al., 2006).

As perdas de nutrientes para o meio aquático influenciam diretamente o desempenho animal, pela redução no aproveitamento dos nutrientes perdidos por lixiviação ou por deteriorização na qualidade da água (FURUYA et al., 1997).

Segundo Vielma et al. (2000), os custos de produção com ração para o salmão-do-atlântico produzido em tanque-rede representam em torno de $50 \%$ para a indústria. Daí a importância da integridade estrutural do pélete durante o manejo e transporte para assegurar menores custos de produção e menos perdas para o meio ambiente. Contudo, apesar dessa importância dada pela indústria aquícola, o insucesso com as partículas extrusadas acabou recebendo uma maior atenção.

O processo de aquecimento controlado propicia aumento da disponibilidade de nutrientes contidos nos ingredientes da ração, o que otimiza a eficiência alimentar, aumenta a digestibilidade, diminui a produção de resíduos e reduz a carga de efluentes que comprometem a qualidade da água

Rev. Acad., Ciênc. Agrár. Ambient., Curitiba, v. 7, n. 4, p. 433-443, out./dez. 2009 
(ALBERNAZ et al., 2000). Em função disso, dietas peletizadas e extrusadas são amplamente preconizadas na alimentação de peixes, principalmente durante os períodos de crescimento e terminação.

Avanços em estudos genéticos de peixes vêm aumentando nos últimos anos e a nutrição depara-se com maiores entraves e limitações para obter uma produção eficiente no crescimento e propagação de novas espécies na aquicultura. Para alcançar uma maximização do desempenho dos peixes, a dieta deve atender às necessidades dos nutrientes essenciais e pró-nutrientes na concentração correta. Uma nutrição equilibrada pode ajudar o peixe a demonstrar todo seu potencial genético no desempenho produtivo e melhor resistência a doenças.

O pacu (Piaractus mesopotamicus Holmberg, 1887) é uma espécie largamente distribuída na bacia dos rios Paraná, Paraguai e Uruguai (GODOY, 1975), onde tem grande importância na pesca comercial, sendo cultivado principalmente nas regiões sul e sudeste e, nos últimos anos, em outros Estados do Brasil (VALENTI et al., 2001). Não existem estudos comparando a eficiência de dietas extrusada e peletizadas testando a levedura Saccharomyces cerevisiae em dietas de peixes nativos, foco deste estudo. Porém, Aarseth et al. (2006) testaram um tipo de levedura vermelha (Xanthophyllomyces dendrorhous) em dietas de salmão e observaram sua capacidade de fermentar açúcares, produzindo altas quantidades de astaxantina como fator pigmentante de sua carne (JOHNSON et al., 1980). A parede celular da $X$. dendrorhous possui uma estrutura composta por polissacarídeos estruturais e proteínas que se assemelha à estrutura da levedura, da qual se extrai o $\beta$-glucano. Segundo esses autores, não houve diferenças na eficiência dessa levedura em relação às temperaturas de extrusão, que foram de $100^{\circ}$ e $140^{\circ} \mathrm{C}$.

Este estudo teve por finalidade testar a eficácia do prebiótico $\beta$-glucano em rações peletizadas e extrusadas no desempenho produtivo de juvenis de pacu ( $P$. mesopotamicus).

\section{MATERIAIS E MÉTODOS}

\section{Local}

O experimento foi conduzido no Laboratório de Peixes Ornamentais do Centro de Aquicultura da UNESP (CAUNESP), em Jaboticabal, São Paulo. A análise bromatológica das dietas foi realizada no Laboratório de Nutrição Animal (LANA), do Departamento de Zootecnia da Faculdade de Ciências Agrárias e Veterinárias da UNESP.

\section{Material biológico e condições ambientais}

Foram utilizados 640 juvenis de pacu (Piaractus m.), com peso inicial de 24,7 \pm 2,0 g, distribuídos em 32 aquários de vidro (130 litros) (20 peixes/caixa). Os aquários foram sifonados diariamente, e a água reposta foi proveniente de poço. O sistema foi provido de aeração contínua e controle de temperatura $\left(26,0 \pm 2,0^{\circ} \mathrm{C}\right)$.

\section{Avaliações físico-químicas da água}

A temperatura média dos aquários foi aferida diariamente com um termômetro de máxima e mínima. As determinações de alcalinidade total $\left(\mathrm{mg}\right.$ de $\left.\mathrm{CaCO}_{3} / \mathrm{L}\right)$ e amônia total $(\mu \mathrm{g} / \mathrm{L})$ foram realizadas no Laboratório Central do CAUNESP. O oxigênio dissolvido $\left(\mathrm{mg} \mathrm{O}_{2} / \mathrm{L}\right)$ e potencial hidrogeniônico $(\mathrm{pH})$ foram medidos quinzenalmente utilizando oxímetro e peagâmetro digital. 


\section{Manejo alimentar, dietas experimentais e delineamento experimental}

Os peixes foram alimentados duas vezes ao dia, às $9 \mathrm{~h}$ e às $17 \mathrm{~h}$. A distribuição de ração foi realizada até não haver mais procura pelos peixes, para evitar sobras. O consumo de alimento foi, dessa forma, registrado diariamente. A formulação e a composição das dietas experimentais são apresentadas na Tabela 1.

TABELA 1 - Formulação e composição percentual e calculada das dietas experimentais

\begin{tabular}{|c|c|c|c|c|}
\hline $\begin{array}{l}\text { Ingredientes } \\
\text { Ingredients }\end{array}$ & Controle (\%) & $\mathrm{T} 1(0,3 \%)$ & $\mathrm{T} 2(0,2 \%)$ & T3 $(0,1 \%)$ \\
\hline $\begin{array}{l}\text { Farelo de Soja } \\
\text { Soybean meal }\end{array}$ & 45,5 & 45,5 & 45,5 & 45,5 \\
\hline $\begin{array}{l}\text { Milho } \\
\text { Corn }\end{array}$ & 22 & 22 & 22 & 22 \\
\hline $\begin{array}{l}\text { Farelo de Trigo } \\
\text { Wheat bran }\end{array}$ & 15 & 15 & 15 & 15 \\
\hline $\begin{array}{l}\text { Farinha de peixe } \\
\text { Fish meal }\end{array}$ & 13 & 13 & 13 & 13 \\
\hline $\begin{array}{l}\text { Bagaço de cana } \\
\text { Sugar cane bagasse }\end{array}$ & 1,5 & 1,2 & 1,3 & 1,4 \\
\hline $\begin{array}{l}\text { Óleo de Soja } \\
\text { Soybean oil }\end{array}$ & 1,5 & 1,5 & 1,5 & 1,5 \\
\hline $\begin{array}{l}\text { Sup. vitamínico mineral }{ }^{1} \\
\text { Viatmin ans Mineral premix }\end{array}$ & 1,5 & 1,5 & 1,5 & 1,5 \\
\hline $\begin{array}{l}\beta \text {-glucano }{ }^{2} \\
\beta \text {-glucan }\end{array}$ & 0 & 0,3 & 0,2 & 0,1 \\
\hline Total & 100 & 100 & 100 & 100 \\
\hline $\begin{array}{l}\text { Composição química } \\
\text { Chemical composition }\end{array}$ & & & \multicolumn{2}{|c|}{$\begin{array}{l}\text { \% de inclusão } \\
\text { \% inclusion }\end{array}$} \\
\hline Matéria Seca & & & \multicolumn{2}{|l|}{89,91} \\
\hline $\begin{array}{l}\text { Dry matter } \\
\text { Proteína bruta } \\
\text { Crude protein }\end{array}$ & & & \multicolumn{2}{|l|}{32,06} \\
\hline $\begin{array}{l}\text { Energia bruta }(\mathrm{kcal} / \mathrm{kg}) \\
\text { Gross energy }\end{array}$ & & & \multicolumn{2}{|l|}{ 4096,03* } \\
\hline $\begin{array}{l}\text { Extrato etéreo } \\
\text { Ether extract }\end{array}$ & & & \multicolumn{2}{|l|}{3,18} \\
\hline $\begin{array}{l}\text { Fibra bruta } \\
\text { Crude fiber }\end{array}$ & & & \multicolumn{2}{|l|}{4,48} \\
\hline $\begin{array}{l}\text { Matéria mineral } \\
\text { Ashes }\end{array}$ & & & \multicolumn{2}{|l|}{7,34} \\
\hline $\begin{array}{l}\text { Extrato não nitrogenado }{ }^{3} \\
\text { Nitrogen free extract }\end{array}$ & & & \multicolumn{2}{|l|}{38,53} \\
\hline
\end{tabular}

Suplemento vitamínico mineral Rovimix peixe: vit. A: 5000.000 UI; vit. D3: 200.000 UI; vit. E: 5.000 UI; vit. K3: 1000 mg; vit. B1: 1500 mg; vit. B2: $1500 \mathrm{mg}$; vit. B6: $1500 \mathrm{mg}$; vit. B12: $4000 \mathrm{mg}$; vit. C: $15000 \mathrm{mg}$; ácido fólico: $500 \mathrm{mg}$; ácido pentotênico: $4000 \mathrm{mg}$; B.H.T.: $12,25 \mathrm{~g}$; biotina: $50 \mathrm{mg}$; inositol: $1000 \mathrm{mg}$; nicotinamida: $7000 \mathrm{mg}$; colina: $40 \mathrm{~g}$; cobalto: $10 \mathrm{mg}$; cobre: $500 \mathrm{mg}$; ferro: $5000 \mathrm{mg}$; iodo $50 \mathrm{mg}$; manganês: $1500 \mathrm{mg}$; selênio: $10 \mathrm{mg}$; zinco: $5000 \mathrm{mg}$; veículo q. s. q. : $1000 \mathrm{mg}$.

2 Nos níveis $0,0,0,1,0,2$ e $0,3 \%$ as diferenças foram preenchidas com elemento inerte.

${ }^{3} \mathrm{ENN}(\%)=\mathrm{MS}(\%)-[\mathrm{PB}(\%)+\mathrm{EE}(\%)+\mathrm{FB}(\%)+\mathrm{MM}(\%)]$.

* Informação em $(\mathrm{Kcal} / \mathrm{kg})$.

Rev. Acad., Ciênc. Agrár. Ambient., Curitiba, v. 7, n. 4, p. 433-443, out./dez. 2009 
As rações peletizadas foram produzidas na Unidade de Preparação de Rações do Centro de Aquicultura da UNESP e na Fábrica de Rações da Faculdade de Ciências Agrárias e Veterinárias da UNESP, campus de Jaboticabal, e as rações extrusadas em uma fábrica de ração comercial. O $\beta$-glucano foi introduzido nas rações antes dos processamentos de peletização e extrusão.

$\mathrm{O}$ experimento foi realizado em um delineamento inteiramente casualizado em esquema fatorial $2 \times 4$, avaliando-se dois processamentos das dietas (extrusadas e peletizadas) com quatro níveis de inclusão do $\beta$-glucano nas rações: controle, $0,1 \%, 0,2 \%$ e $0,3 \%$. Cada tratamento constou de quatro repetições. $O$ aditivo $\beta$-glucano utilizado é um prebiótico rico em $\beta$-glucanos, extraídos da levedura Saccharomyces cerecisiae de cepas selecionadas. Esse prebiótico foi adquirido comercialmente, e possui pureza de $85 \%$.

\section{Avaliação dos parâmetros zootécnicos}

Para avaliar o desempenho dos peixes, esses foram pesados e medidos do início do experimento até os 30,60 e 90 dias, e esses dados podem ser observados na Tabela 3, na qual foi realizada uma média de todos esses valores durante o período experimental de 90 dias.

O ganho de peso dos peixes (GP) foi calculado por:

$$
G P(g)=\frac{\text { Peso Médio Final }- \text { Peso Médio Inicial }}{\text { Tempo de Experimento }(\text { dias })}
$$

Para determinação da taxa de crescimento específico (TCE) foi empregada a seguinte equação:

$$
\operatorname{TCE}(\% / \text { dia })=\frac{(\ln ) \text { Peso Total Final }-(\ln ) \text { Peso Total Inicial } x 100}{\text { Tempo de Experimento }(\text { dias })}
$$

O consumo total de ração foi determinado por:

$$
\text { Consumo }=\frac{\text { Ração Pesada no Início }- \text { Sobras do Final }}{\text { Tempo do Experimento }(\text { dias })}
$$

A conversão alimentar aparente (CA) foi obtida a partir da seguinte equação:

$$
C A=\frac{\text { Consumo Total de Ração }(g)}{[\text { Ganho de Peso dos Peixes }(g)] x[\text { Número de Peixes na Parcela }]}
$$




\section{Análise estatística}

O experimento foi em delineamento inteiramente casualizado em esquema fatorial com duas dietas (extrusada e peletizada) e quatro níveis de inclusão do $\beta$-glucano. Os dados obtidos foram submetidos à análise de variância (teste $\mathrm{F}$ ) e, quando detectadas diferenças, as médias \pm desvio padrão das médias foram comparados pelo teste de Duncan ao nível de 5\% de confiabilidade. As análises estatísticas foram realizadas utilizando o programa estatístico SAS 9.0.

\section{RESULTADOS}

\section{Parâmetros físico-químicos da água} na Tabela 2.

Os parâmetros físicos e químicos foram mensurados aleatoriamente por aquário e descritos

TABELA 2 - Valores médios \pm desvio padrão e coeficiente de variação dos parâmetros físicos e químicos da água dos aquários de juvenis de pacu

\begin{tabular}{lcc}
\hline & $\begin{array}{c}\text { Tratamentos } \\
\text { Treatments }\end{array}$ & $\begin{array}{c}\text { CV\% } \\
\text { Variance coefficient }\end{array}$ \\
\hline $\begin{array}{l}\text { Temperatura }\left({ }^{\circ} \mathrm{C}\right) \\
\text { Temperature }\end{array}$ & $26,25 \pm 0,92$ & 1,02 \\
$\begin{array}{l}\text { Alcalinidade }(\mathrm{mg} / \mathrm{L}) \\
\text { Alcalinity } \\
\text { Amônia Total }(\mu \mathrm{g} / \mathrm{L})\end{array}$ & $82,4 \pm 4,25$ & 3,33 \\
$\begin{array}{l}\text { Ammonia } \\
\text { Oxigênio dissolvido }(\mathrm{mg} / \mathrm{L})\end{array}$ & $8,87 \pm 1,34$ & 2,57 \\
$\begin{array}{l}\text { Dissolved oxygen } \\
\text { pH }\end{array}$ & $3,52 \pm 1,68$ & 5,87 \\
$p H$ & $7,74 \pm 0,27$ & 3,66 \\
\hline
\end{tabular}

\section{Desempenho zootécnico}

De acordo com os resultados apresentados na Tabela 3, não foram observadas diferenças significativas em relação ao ganho de peso pelos processamentos com a ração extrusada e peletizada. $\mathrm{O}$ peso final também não foi influenciado pelos processamentos.

O nível $0,3 \%$ de inclusão do prebiótico proporcionou, no último período experimental, um ganho de peso de 10,5\% superior ao grupo controle. Apesar de não haver diferenças significativas entre os resultados de ganho de peso entre os processamentos das rações, a ração peletizada $(43,36 \mathrm{~g})$ mostrou melhores resultados de desempenho produtivo que a ração extrusada $(42,79 \mathrm{~g})$.

A mesma tendência ocorreu com o peso final, em que o maior nível de inclusão do $\beta$-glucano induziu a um crescimento $5 \%$ superior dos peixes que não receberam o prebiótico na dieta. Porém, estatisticamente não houve diferenças significativas entre os tratamentos.

Não ocorreram diferenças significativas na taxa de crescimento específico dos peixes em relação ao processamento das rações e aos níveis de inclusão do $\beta$-glucano nas dietas. Entretanto, apesar de não significativo, a maior taxa de crescimento específico foi observada para o tratamento com $0,3 \%$ de inclusão.

Rev. Acad., Ciênc. Agrár. Ambient., Curitiba, v. 7, n. 4, p. 433-443, out./dez. 2009 
No presente estudo, os peixes não apresentaram diferença de consumo entre as rações peletizada e extrusada. Entretanto, o consumo da ração extrusada foi superior em relação à ração peletizada. O nível $0,3 \%$ de $\beta$-glucano na dieta proporcionou maior consumo.

Os processamentos das rações avaliadas proporcionaram diferenças na conversão alimentar dos peixes, apesar de não significativo. Entretanto, a ração extrusada apresentou pior conversão em relação à ração peletizada.

TABELA 3 - Valores de F, coeficiente de variação (C.V.) e médias obtidas para ganho de peso (G.P.), peso final

(P.F.), taxa de crescimento específico (T.C.E.), consumo e conversão alimentar (C.A.) para juvenis de pacu

\begin{tabular}{|c|c|c|c|c|c|c|}
\hline & \multirow[t]{2}{*}{$\begin{array}{l}\text { Estatística } \\
\text { Statistics }\end{array}$} & \multicolumn{5}{|c|}{$\begin{array}{l}\text { Período Experimental }(0-90 \text { dias }) \\
\text { Experimental period ( } 0-90 \text { days })\end{array}$} \\
\hline & & $\begin{array}{l}\text { G.P. } \\
B . W .\end{array}$ & $\begin{array}{l}\text { P. F. } \\
\text { F.W. }\end{array}$ & $\begin{array}{l}\text { T.C.E. } \\
\text { S.G.R. }\end{array}$ & $\begin{array}{c}\text { CONS } \\
\text { CONSUMPTION }\end{array}$ & $\begin{array}{l}\text { C.A. } \\
\text { F.C. }\end{array}$ \\
\hline \multirow{4}{*}{$\begin{array}{l}\text { Valores para } \mathrm{F} \\
\text { F values }\end{array}$} & $\begin{array}{c}\text { Processamento }(\mathrm{P}) \\
\text { Processment }\end{array}$ & $0,86 \mathrm{~ns}$ & $0,79 \mathrm{~ns}$ & $0,30 \mathrm{~ns}$ & $0,05 \mathrm{~ns}$ & $41 \mathrm{~ns}$ \\
\hline & $\begin{array}{l}\text { Níveis }(\mathrm{N}) \\
\text { Levels }\end{array}$ & $0,72 \mathrm{~ns}$ & $0,1 \mathrm{~ns}$ & $0,13 \mathrm{~ns}$ & $0,07 \mathrm{~ns}$ & $0,1 \mathrm{~ns}$ \\
\hline & $\begin{array}{l}\text { Interação }(\mathrm{P} \times \mathrm{N}) \\
\text { Interactions }\end{array}$ & $0,32 \mathrm{~ns}$ & $1,71 \mathrm{~ns}$ & $0,59 \mathrm{~ns}$ & $0,54 \mathrm{~ns}$ & $39 \mathrm{~ns}$ \\
\hline & C.V. & 21,58 & 0,14 & 17,76 & 22,83 & 23,7 \\
\hline \multirow{2}{*}{$\begin{array}{l}\text { Médias Processamento } \\
\text { Processment Means }\end{array}$} & $\begin{array}{l}\text { Ração Extrusada } \\
\text { Extruded diet }\end{array}$ & 42,79 & 51,0 & 1,95 & 141,77 & 3,02 \\
\hline & $\begin{array}{l}\text { Ração Peletizada } \\
\text { Pelletized diet }\end{array}$ & 43,36 & 52,03 & 1,82 & 120,68 & 2,82 \\
\hline \multirow{4}{*}{$\begin{array}{l}\text { Médias Níveis } \\
\text { Levels Means }\end{array}$} & $\begin{array}{l}\text { Controle }(0 \%) \\
\text { Control }\end{array}$ & 41,05 & 52,0 & 1,85 & 113,64 & 2,43 \\
\hline & $0,30 \%$ & 45,87 & 54,7 & 2,14 & 153,2 & 3,18 \\
\hline & $0,20 \%$ & 41,68 & 48,33 & 1,76 & 135,32 & 3,23 \\
\hline & $0,10 \%$ & 43,7 & 52,75 & 1,8 & 122,75 & 2,84 \\
\hline
\end{tabular}

\section{DISCUSSÃO}

\section{Parâmetros físicos e químicos da água}

Os resultados demonstraram que a qualidade da água dos aquários não foi alterada, sendo que os parâmetros avaliados permaneceram de acordo com os padrões recomendados por Sipaúba-Tavares (1995) e Kubitza (2000). Em relação ao oxigênio dissolvido, a tolerância para muitas espécies de peixes é de 1 a $9 \mathrm{mg} / \mathrm{L}$, sendo que a faixa ideal exigida pelas espécies está relacionada com a fase de crescimento e reprodução, embora a maior parte das espécies tenham uma exigência acima de $5 \mathrm{mg} / \mathrm{L}$ (BOYD, 1982).

\section{Desempenho zootécnico}

Pelo fato do nível $0,3 \%$ de inclusão do prebiótico proporcionar um ganho de peso 10,5\% superior ao grupo controle, esse índice, em uma produção de larga escala de peixes, pode representar um impacto econômico significativo na atividade produtiva.

Rev. Acad., Ciênc. Agrár. Ambient., Curitiba, v. 7, n. 4, p. 433-443, out./dez. 2009 
Apesar de não haver diferenças significativas nos resultados de ganho de peso entre os processamentos das rações, a ração extrusada mostrou-se menos eficiente em relação à peletizada. Avaliando diferentes processamentos de ração para o acará-bandeira (Pterophilum scalare), Rodrigues e Fernandes (2006) obtiveram resultados semelhantes aos desse experimento, em que a ração peletizada e extrusada não apresentou diferenças significativas no desempenho produtivo.

Neste estudo, a ração extrusada apresentou maior conversão em relação à ração peletizada, tal como o estudo de Pezzato et al. (2002), avaliando processamento de dietas para tilápias-do-nilo (Oreochromis niloticus) observaram uma melhor conversão alimentar nos tratamentos com a ração extrusada, além de melhor desempenho produtivo, quando comparado à ração peletizada. De acordo com a literatura as partículas que compõem o pélete extrusado apresentam melhores respostas de digestibilidade, o que indica uma tendência de melhora na disponibilidade dos nutrientes contidos nas dietas, em que a adição de calor e umidade altera os componentes da mistura com o amido e a proteína (THOMAS; VAN DER POEL, 1996), melhorando a biodisponibilidade dos ingredientes (HILTON et al., 1981). Furuya et al. (1997) também não encontraram diferenças significativas entre os processamentos, mas concluíram que a ração peletizada proporciona melhores resultados econômicos para a tilápia-donilo na fase de terminação.

Efthimiou (1996), em dietas com $\beta$-glucano para o sea bass, não observou diferenças significativas no ganho de peso. Hardie et al. (1991) e Hardie et al. (1990), que administraram oralmente esse imunoestimulante em dentex (Dentex dentex) e salmão-do-atlântico (Salmo salar), também não observaram diferença no ganho de peso dos peixes alimentados com diferentes níveis de $\beta$-glucano em relação ao grupo controle. Resultados contraditórios foram obtidos em estudo realizado por Ai et al. (2007), no qual o aumento no ganho de peso do yellow croaker (Psendosciaena crocea) foi atribuído ao uso de dosagens baixas de glucano $(0,09 \% / \mathrm{kg}$ de ração) durante oito semanas. Contudo, o crescimento não foi favorecido quando esses autores utilizaram dosagem maior $(0,18 \%)$ de $\beta$-glucano, sugerindo que o uso de baixas dosagens fornecem uma suplementação adequada para otimizar o ganho de peso dessa espécie.

Autores como Wigglesworth e Griffith (1994) observaram em camarões (Penaeus monodon) uma alta capacidade de digestão do $\beta$-glucano, sugerindo que o ganho de peso aumentou pelos benefícios energéticos através deste prebiótico. Para peixes não existem evidências a respeito e os mecanismos que promovem seu crescimento pela ação do $\beta$-glucano ainda não foram completamente identificados (AI et al., 2007). Porém, segundo Lopéz et al. (2003), esse aumento no ganho de peso pode estar relacionado com a provável degradação do $\beta$-glucano pela glucanase, produzindo energia, permitindo o uso de mais proteínas e favorecendo o crescimento.

Misra et al. (2006), avaliando o $\beta$-glucano no Labeo rohita, observaram aumento da taxa de crescimento específico (TCE) nos tratamentos com inclusão de 0,25\% e 0,5\%. Contraditoriamente, Ai et al. (2007), testando níveis de $\beta$-glucano na dieta do yellow croaker ( $P$. crocea), observaram uma TCE significativamente maior nos peixes do tratamento com $0,09 \%$ de inclusão deste prebiótico em comparação com o tratamento controle. Porém, ao nível de $0,18 \%$ de $\beta$-glucano a TCE não se diferenciou do grupo controle. Cook et al. (2003), em estudos com o snapper (Pagrus auratus, Sparidae), e Hidalgo et al. (2006), alimentando juvenis de dentex (Dentex dentex), não notaram diferenças significativas na TCE dos animais alimentados com prebiótico quando comparado ao grupo controle. Resultados semelhantes são observados com o salmão-do-atlântico, em que não houve efeito do prebiótico no crescimento (GILDBERG et al., 1995).

O emprego do $\beta$-glucano não proporcionou aumento no consumo dos peixes alimentados com rações contendo o prebiótico na sua composição, provavelmente pela razão dos níveis de inclusão serem modestos, não alterando de forma significativa as características do alimento de todos os tratamentos.

Misra et al. (2006), testando níveis de $\beta$-glucano de $0,1 \%, 0,25 \%$ e $0,5 \%$ para juvenis de Labeo rohita, não observaram diferença significativa entre os níveis do imunoestimulante; porém, o tratamento com o nível superior apresentou melhor conversão alimentar em relação aos outros tratamentos num período de 56 dias.

Bagni et al. (2005) observaram conversões alimentares que variaram de 0,7 a 5,0, no bagredo-canal (Dicentrarchus labrax), após administração com $\beta$-glucano em níveis semelhantes ao desse.

Rev. Acad., Ciênc. Agrár. Ambient., Curitiba, v. 7, n. 4, p. 433-443, out./dez. 2009 
Misra et al. (2006), administrando oralmente o $\beta$-glucano na alimentação do Labeo robita, obtiveram valores de conversão alimentar de 2,0 a 3,5.

A ausência de diferenças significativas no desempenho dos animais pode ser devido ao curto período de tempo em que foi realizado este trabalho. Dessa forma, outros estudos com maior tempo de duração podem possibilitar um melhor entendimento e importância desse imunoestimulante na alimentação de juvenis de pacu.

\section{CONCLUSÃO}

Os resultados obtidos mostram que o desempenho produtivo de juvenis de pacu não foi afetado pelos níveis de $\beta$-glucano e pelo tipo de processamento da ração.

\section{AGRADECIMENTOS}

À FAPESP, pelo auxílio-bolsa e reserva técnica à primeira autora deste artigo.

\section{REFERÊNCIAS}

AARSETH, K. A. et al. Effects of red yeast inclusions in diets for salmonids and extrusion temperature on pellet tensile strength: weilbull analysis. Animal Feed Science and Technology, v. 126 , n. $1 / 2$, p. $75-91,2006$.

AI, Q. et al. Effects of dietary $\beta-1,3$ glucan on innate immune response of large yellow croaker Pseudosciana crocea. Fish and Shellfish Immunology, Aberdeen, v. 22, n. 4, p. 394-402, 2007.

ALBERNAZ, N. S. et al. Efeito do processamento da ração sobre os valores de digestibilidade dos nutrientes para piau verdadeiro (Leporinus elongatus) In: REUNIÃO ANUAL DA SOCIEDADE BRASILEIRA DE ZOOTECNIA - SBZ, 37., 2000, Viçosa. Anais... Viçosa: SBZ, 2000. p. 282.

ANDRIGUETO, J. M. et al. Nutrição animal: as bases e os fundamentos da nutrição animal os alimentos - os alimentos. 4. ed. São Paulo: Nobel, 1981. 395 p.

BAGNI, M. et al. Short and long-term effects of a dietary yeast $\beta$-glucan (Macrogard) and alginic acid (Ergosan) preparation on immune response in sea bass (Dicentrarchus labrax). Fish and Shellfish Immunology, Aberdeen, v. 18, n. 4, p. 311-325, 2005.

BOYD, C. E. Water quality management for pond fish culture. Developments in Aquaculture and Fisheries Science, v. 9, p. 318, 1982.

CAMARGO, S. G. O.; POUEY, J. L. O. F. Aqüicultura: um mercado em expansão. Revista Brasileira de Agrociências, Pelotas, v. 11, n. 4, p. 393-396, 2005.

COOK, M. T. et al. Administration of a commercial immunostimulant preparation, EcoActiva ${ }^{\mathrm{TM}}$ as a feed supplement enhances macrophages respiratory burst and the growth rate of snapper (Pagrus auratus, Sparidae (Bloch and Schneider)) in winter. Fish and Shellfish Immunology, Aberdeen, v. 14, n. 4, p. 333-345, 2003.

EFTHIMIOU, S. Dietary intake of $\beta-1,3 / 1,6$ glucans in juvenile dentex (Dentex dentex). Sparidae: effect on growth performance, mortalities and non-specific defense mechanisms. Journal of Applied Ichthyology, Hoboken, v. 12, n. 1, p. 1-7, 1996. 
FOOD AND AGRICULTURE ORGANIZATION OF THE UNITED NATIONS - FAO. Review of the state of world Aquaculture. Roma: FAO, 2003. (Fisheries Circular, 886 - Revision 2).

FURUYA, V. R. B. et al. Farelo de canola na alimentação da tilápia do Nilo (Oreochromis niloticus L.), durante o período de reversão de sexo. Revista Brasileira de Zootecnia, Viçosa, v. 26, n. 6, p. 1067-1073, 1997.

GILDBERG, A. et al. Growth and survival of Atlantic salmon (Salmo salar) fry given diets supplemented with fish protein hydrolysate and lactic acid bacteria during a challenge trial with Aeromonas salmonicida. Aquaculture, Amsterdam, v. 138, n. 1/4, p. 23-24, 1995.

GODOY, M. P. Peixes do Brasil. Piracicaba: Franciscana, 1975.

HARDIE L. A. et al. Cyclostratigraphy and dolomitization of the middle triassic latemar buildup, the dolomites, northern Italy. In: DOLOMIEU CONFERENCE ON CARBONATE PLATFORMS AND DOLOMITIZATION, 1991, St. Ulrich. Abstract... St. Ulrich: Guidebook Excursion, 1991. p. 56.

HARDIE, L. J. et al. The effect of vitamin e on the immune response of Atlantic salmon (Salmo salar). Aquaculture, Amsterdam, v. 87, n. 1, p. 1-13, 1990.

HIDALGO, A. et al. Neurotrophic and gliatrophic contexts in drosophila. Brain Behavior and Evolution, Orlando, v. 68, n. 3, p. 173-180, 2006.

HILTON, J. W. et al. Effect of extrusion processing and steam pelleting diets on pellet durability, pellet water absorption, and the physiological response of rainbow trout (Salmo gairdneri R.). Aquaculture, Amsterdam, v. 25, n. 2/3, p. 185-194, 1981.

JOHNSON, E. A. et al. Phaffia rhodosyma as an astaxanthin source in salmonid diets. Aquaculture, Amsterdam, v. 20, n. 2, p.123-134, 1980.

KUBITZA, F. Tilápia: tecnologia e planejamento na produção comercial. Jundiaí: Acqua Imagem, 2000.

LOPÉZ, N. et al. Phisiological, nutritional, and immunological role of dietary $\beta$ 1-3 glucan and ascorbic acid 2-monophospahte in Litopenaeus vannamei juvenilles. Aquaculture, Amsterdam, v. 224 , n. 1-4, p. 223-243, 2003.

MISRA, K. M. et al. Effect of long term administration of dietary $\beta$-glucan on immunity, growth and survival of Labeo robita fingerlings. Aquaculture, Amsterdam, v. 255, n. 1, p. 84-94, 2006.

PEZZATO, L. E. et al. Diâmetro do ingrediente e a digestibilidade aparente de rações de duas espécies de peixes tropicais. Acta Scientiarum: Animal Sciences, Maringá, v. 24, n. 4, p. 901-907, 2002.

PRIETO, M. J. et al. Tipo de alevino, sobrevivência e desempenho inicial de pós-larvas de pacu (Piaractus mesopotamicus). Ciência e Agrotecnologia, Lavras, v. 30, n. 5, p. 1002-1007, 2006.

RODRÍGUES, L. A.; FERNANDES, J. B. K. Influência do processamento da dieta no desempenho produtivo do acará-bandeira (Pterophyllum scalare). Acta Scientiarium: Animal Sciences, Maringá, v. 28 , n. 1 , p. $113-119,2006$

SAS INSTITUTE. User's guide: statistics, Software SAS 9.0. Cary: SAS Inst. Inc., 1998.

SIPAÚBA-TAVARES, L. H. Limnologia aplicada à aqüicultura. Jaboticabal: FUNEP, 1995.

Rev. Acad., Ciênc. Agrár. Ambient., Curitiba, v. 7, n. 4, p. 433-443, out./dez. 2009 
THOMAS, N.; VAN DER POEL, A. F. B. Physical quality of pelleted animal feed 1. Criteria for pellet quality. Animal Feed Science Technology, Shannon, v. 61, n. 1/4, p. 89-112, 1996.

VALENTI, L. M. P. et al. Feed intake and growth of fast and slow growing strains of rainbow trout (Oncorbynchus mykiss) fed by automatic feeders or by self-feeders. Aquaculture, Amsterdam, v. 195 , n. $1 / 2$, p. $121-131,2001$.

VIEIRA, J. S. et al. Efeito do preocessamento do milho sobre o desempenho e composição de carcaça de piaba (Leporinus friderici) criada em tanques-rede. Ciência Agrotecnológica, Lavras, v. 29, n. 2 , p. $453-458,2005$.

VIELMA, J. et al. Influence of dietary soy and phytase levels on performance and body composition of large rainbow trout (Oncorbynchus mykiss) and algal availability of phosphorus load. Aquaculture, Amsterdam, v. 183, n. 3/4, p. 349-362, 2000.

WIGGLESWORTH, J. M.; GRIFFITH, D. R. W. Carbohydrate digestion in Peaneus monodon. Marine Biology, Berlin, v. 120, n. 4, p. 571, 1994.

Recebido: 15/08/2008

Received: 08/15/2008

Aprovado: 04/05/2009

Approved: 05/04/2009

Rev. Acad., Ciênc. Agrár. Ambient., Curitiba, v. 7, n. 4, p. 433-443, out./dez. 2009 